treatments: correlation with repair. NMR Biomed. 1998;11(1):1-10.

4. Sacco A, Doyonnas R, Kraft P, Vitorovic S, Blau HM. Self-renewal and expansion of single transplanted muscle stem cells. Nature. 2008; 456(7221):502-506.

5. Maguire KK, Lim L, Speedy S, Rando TA Assessment of disease activity in muscular dystrophies by noninvasive imaging. $J$ Clin Invest. 2013;123(5):2298-2305.

6. Nishijo K, et al. Biomarker system for studying muscle, stem cells, and cancer in vivo. FASEB J. 2009;23(8):2681-2690.
7. Bittner RE, et al. Dysferlin deletion in SJL mice (SJLDysf) defines a natural model for limb girdle muscular dystrophy 2B. Nat Genet. 1999;23(2):141-142.

8. Weller AH, Magliato SA, Bell KP, Rosenberg NL. Spontaneous myopathy in the SJL/J mouse: pathology and strength loss. Muscle Nerve. 1997; 20(1):72-82.

9. Han R, Campbell KP. Dysferlin and muscle membrane repair. Curr Opin Cell Biol. 2007;19(4):409-416.

10. Hornsey MA, Laval SH, Barresi R, Lochmüller $\mathrm{H}$, Bushby K. Muscular dystrophy in dysferlin-deficient mouse models. Neuromuscul Disord. 2013;23(5):377-387.
11. Ho M, et al. Disruption of muscle membrane and phenotype divergence in two novel mouse models of dysferlin deficiency. Hum Mol Genet. 2004;13(18):1999-2010.

12. Bansal D, et al. Defective membrane repair in dysferlin-deficient muscular dystrophy. Nature. 2003;423(6936):168-172.

13. Stedman HH, et al. The mdx mouse diaphragm reproduces the degenerative changes of Duchenne muscular dystrophy. Nature. 1991;352(6335):536-539.

14. Wallace GQ, McNally EM. Mechanisms of muscle degeneration, regeneration, and repair in the muscular dystrophies. Annu Rev Physiol. 2009;71:37-57.

\title{
Where hypertension happens
}

\author{
Timothy L. Reudelhuber
}

\begin{abstract}
Laboratory of Molecular Biochemistry of Hypertension, Clinical Research Institute of Montreal, Division of Experimental Medicine, Department of Medicine, McGill University, Montreal, Quebec, Canada. Department of Medicine, University of Montreal, Montreal, Quebec, Canada.
\end{abstract}

\begin{abstract}
Essential hypertension, which accounts for $\mathbf{9 0 \%}-\mathbf{9 5 \%}$ of all cases of hypertension seen in the clinic, is also referred to as idiopathic hypertension, because we simply don't understand the cause(s). Although many theories have been advanced, in the current issue of the JCI, Gonzalez-Villalobos et al. present further evidence implicating the intrarenal renin-angiotensin system and take us one step further by proposing a mechanism underlying this pathology.
\end{abstract}

We know a lot about the environmental triggers for essential hypertension; there's no doubt that aging, obesity associated with increased insulin resistance, excessive alcohol intake, ethnicity, stress, and (in certain individuals) excessive dietary salt intake or inadequate dietary potassium or calcium can contribute to high blood pressure (1). Nevertheless, we don't have a clear picture of what happens in the body to translate most of these stimuli into a pathological condition. Any attendee of a recent hypertension research meeting will know that there is no shortage of hypotheses, including increased production of reactive oxygen species, enhanced reactivity of resistance blood vessels to vasoconstriction, vascular inflammation involving immune cells, and malfunctioning baroreceptors or triggers in the central nervous system. Although animal models exist in which each of these mechanisms can be shown to alter blood pressure and each may indeed contribute to a greater or lesser degree to elevating blood pres-

Conflict of interest: The author has declared that no conflict of interest exists.

Citation for this article: J Clin Invest. 2013; 123(5):1934-1936. doi:10.1172/JCI69296. sure in hypertensive patients, many lines of evidence suggest that it's in the kidney that it all comes together.

\section{The kidney as a critical hypertension locus}

Several researchers have demonstrated that transplanting the kidneys of hypertensive rats into normotensive control rats rendered the recipients hypertensive (2-4). A molecular-era refinement of such transplant experiments suggests that the hypertension that travels with the kidney might be due in part to the renin-angiotensin system (RAS). In the classical RAS, angiotensinogen from the liver is cleaved by kidney-derived renin to form angiotensin I, which is subsequently cleaved by angiotensinconverting enzyme (ACE) present on the surface of endothelial cells throughout the body to generate the potent vasopeptide angiotensin II (Figure 1A). There are two angiotensin II receptors, and it is the angiotensin II receptor type $1\left(\mathrm{AT}_{\mathrm{R}}\right)$ subtype that increases blood pressure and fluid volume through vasoconstriction and stimulation of aldosterone secretion from the adrenal gland and salt retention by the kidneys. Coffman and colleagues transplanted the kidneys of $A T 1_{R}$-defi- cient mice into control animals and vice versa (5). Subsequent infusion of these mice for two weeks with a high dose of angiotensin II revealed that the mice with AT $1_{R}$ expression in the kidney, but a complete lack of this receptor elsewhere in the body, developed hypertension and cardiac hypertrophy. Surprisingly, the mice that lacked $\mathrm{AT} 1_{\mathrm{R}}$ in the kidney developed neither hypertension nor cardiac hypertrophy, despite expressing AT $1_{\mathrm{R}}$ everywhere else in the body. The overall implication of these studies is that angiotensin II sensing within the kidney is a critical mediator of hypertension.

However, a model in which circulating angiotensin II causes hypertension by a direct action on the kidney fails to explain a long-standing clinical conundrum: how does ACE inhibition remain effective in lowering blood pressure in individuals whose circulating angiotensin II gradually returns to pretreatment levels (socalled angiotensin escape) (6)? In this issue of the JCI, Gonzalez-Villalobos et al. address this question by investigating the role of the intrarenal RAS in hypertension by inactivating the Ace gene in mouse kidney (7). The way that they achieved this was somewhat unconventional. They began with mice that had a whole-body inactivation of Ace. However, because the complete inactivation of any of the RAS genes, including Ace, results in severe renal defects and high neonatal lethality in both humans and mice (8), Gonzalez-Villalobos et al. generated Ace knockout mice that ectopically expressed ACE in either hepatocytes (ACE $3 / 3$ mice) 
A

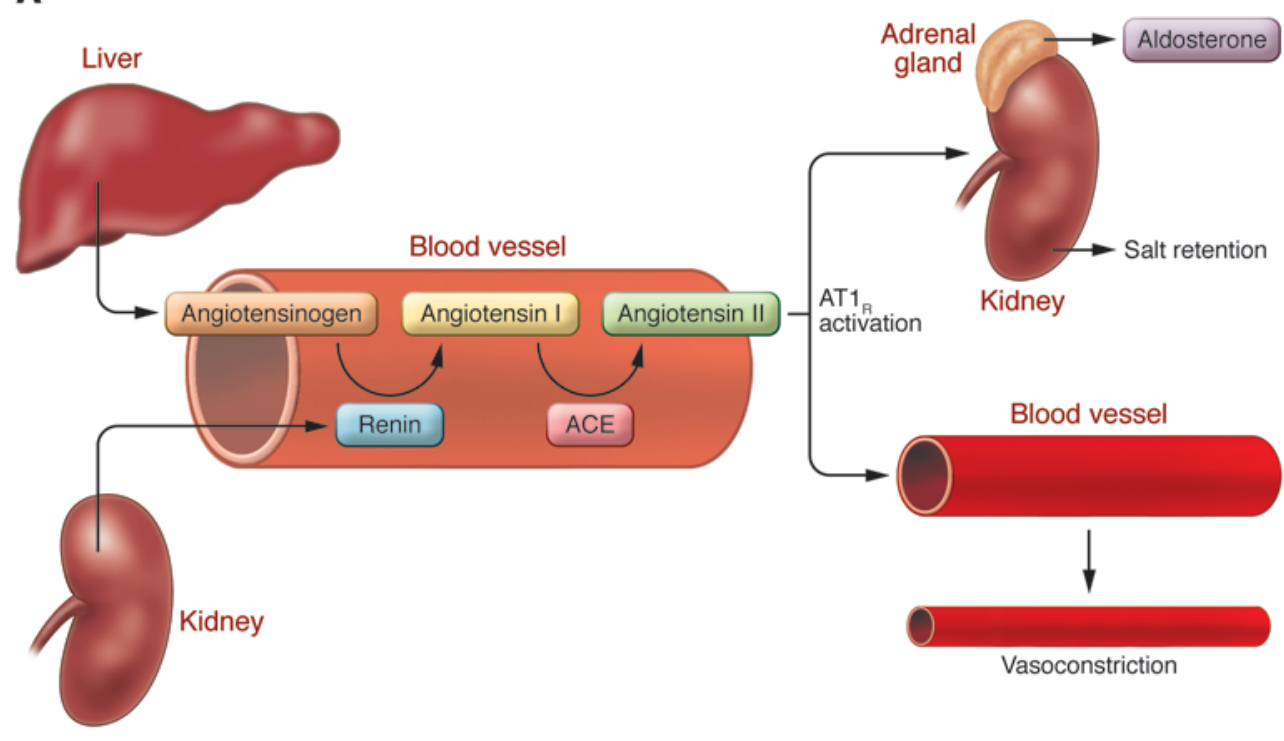

B

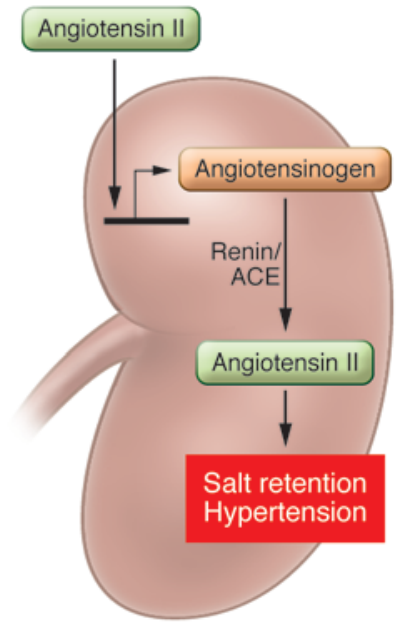

Figure 1

The circulating and intrarenal RAS. (A) Angiotensin II is produced in the circulation and can raise blood pressure by promoting vasoconstriction and increasing salt and water retention. (B) Gonzalez-Villalobos et al. (7) suggest that circulating angiotensin II stimulates an intrarenal RAS cascade and that the resulting locally produced angiotensin II causes hypertension.

or myelomonocytic cells (ACE 10/10 mice). According to the authors, this ectopically expressed ACE prevents all of the renal developmental defects and makes it possible to study mice that lack ACE in the kidney. Surprisingly, both lines of mice displayed a blunted hypertensive response to infused angiotensin II, although they have normal expression of $\mathrm{AT} 1_{\mathrm{R}}$ in the kidney. The authors propose that circulating angiotensin II induces expression of angiotensinogen in the kidney, which itself gets converted by locally produced renin and ACE to angiotensin II (Figure 1B). The authors argue that it is this locally produced angiotensin II that affects salt retention in the tubules and results in hypertension. Indeed, the ACE 10/10 mice fail to induce the expression and activation of sodium transporters in response to the angiotensin infusion (7).

\section{Whole-body context}

This model provides an attractive way to explain the phenomenon of angiotensin escape mentioned above. However, there are some caveats. First, these mice also fail to mount a hypertensive response to nitric oxide synthesis inhibition, which doesn't clearly implicate the renal RAS and which suggests that a pathway other than the renal RAS may also be affected. Second, unlike that in the control mice, renal angiotensinogen expression is not induced in the ACE 10/10 mice in response to the angiotensin II infusion (7). Although this is presented as further evidence of the importance of the intrarenal RAS, it's difficult to envision the mechanism whereby the absence of ACE in the kidney would affect the expression of kidney angiotensinogen. In fact, a recent report from Matsusaka et al. (9) demonstrates that the angiotensin II produced in the kidney is derived from angiotensinogen produced by the liver, so the actual contribution of kidney-produced angiotensinogen is not entirely clear. Finally, the elephant in the room is the fact that the ACE 10/10 mice used for the bulk of these studies lack ACE enzyme not only in the kidney, but everywhere else in the body with the exception of myeloid cells. As a result, it is impossible to rule out the contribution of ACE deficiency in another tissue to the observations. For example, the activity of the RAS in the brain has been shown to control thirst, the ability to concentrate urine, and sympathetic outflow (reviewed in ref. 10), and it's easy to see how any of these these might impact hypertension. The brain-kidney link has important clinical significance as well: denervation of kidneys in rats has the ability to reduce blood pressure and modulate salt bal- ance in hypertensive rats (11) and is being tested in clinical trials for the treatment of resistant hypertension (12). Thus, the role of the brain and perhaps other ACEexpressing tissues in the response of ACE 10/10 mice to angiotensin II might warrant more attention.

\section{Conclusions}

Overall, the finding that removing ACE activity in tissues can reduce the effects of angiotensin II on hypertension and salt balance is both surprising and potentially important. It reminds us that the circulating RAS, which is still the system we teach primarily to students, may not play the lead role in hypertension. In addition, while the assignment of the kidney as the nexus of hypertension is more convincing when taking into account the previous transplantation studies, it may be too soon to rule out other players.

\section{Acknowledgments}

The author's research is supported by a grant from the Canadian Institutes for Health Research (MOP-106632).

Address correspondence to: Timothy L. Reudelhuber, 110 Pine Avenue West, Montreal, PQ H2W1R7, Canada. Phone: 514.987.5716; E-mail: Tim.Reudelhuber@ ircm.qc.ca. 
1. Chobanian AV, et al. The Seventh Report of the Joint National Committee on Prevention, Detection, Evaluation, and Treatment of High Blood Pressure: the JNC 7 report. JAMA. 2003;289(19):2560-2572.

2. Dahl LK, Heine M. Primary role of renal homografts in setting chronic blood pressure levels in rats. Circ Res. 1975;36(6):692-696.

3. Bianchi G, Fox U, Di Francesco GF, Giovanetti AM, Pagetti D. Blood pressure changes produced by kidney cross-transplantation between spontaneously hypertensive rats and normotensive rats. Clin Sci Mol Med. 1974;47(5):435-448.

4. Grisk $\mathrm{O}$, et al. Long-term arterial pressure in spontaneously hypertensive rats is set by the kidney.
J Hypertens. 2002;20(1):131-138.

5. Crowley SD, et al. Angiotensin II causes hypertension and cardiac hypertrophy through its receptors in the kidney. Proc Natl Acad Sci U S A. 2006;103(47):17985-17990

6. Juillerat L, et al. Determinants of angiotensin II generation during converting enzyme inhibition. Hypertension. 1990;16(5):564-572.

7. Gonzalez-Villalobos RA, et al. The absence of intrarenal ACE protects against hypertension. J Clin Invest. 2013;123(5):2011-2023.

8. Gribouval O, et al. Spectrum of mutations in the renin-angiotensin system genes in autosomal recessive renal tubular dysgenesis. Hum Mutat.
2012;33(2):316-326

9. Matsusaka T, et al. Liver angiotensinogen is the primary source of renal angiotensin II. J Am Soc Nephrol. 2012;23(7):1181-1189.

10. Marc Y, Llorens-Cortes C. The role of the brain renin-angiotensin system in hypertension: implications for new treatment. Prog Neurobiol. 2011;95(2):89-103.

11. Foss JD, Fink GD, Osborn JW. Reversal of genetic salt-sensitive hypertension by targeted sympathetic ablation. Hypertension. 2013;61(4):806-811.

12. Persu A, Renkin J, Thijs L, Staessen JA. Renal denervation: ultima ratio or standard in treatment-resistant hypertension. Hypertension. 2012;60(3):596-606.

\title{
Anonymous sources: where do adult $\beta$ cells come from?
}

\author{
Michael S. German
}

Diabetes Center, Eli and Edythe Broad Center of Regeneration Medicine and Stem Cell Research, and Department of Medicine, UCSF, San Francisco, California, USA.

\begin{abstract}
Evidence that the pool of insulin-producing $\beta$ cells in the pancreas is reduced in both major forms of diabetes mellitus has led to efforts to understand $\beta$ cell turnover in the adult pancreas. Unfortunately, previous studies have reached opposing conclusions regarding the source of new $\beta$ cells during regeneration in the adult pancreas. In this issue of the JCI, Xiao et al. use a novel mouse model for detecting new $\beta$ cells derived from non- $\beta$ cells to demonstrate the absence of $\beta$ cell neogenesis from non- $\beta$ cells during normal postnatal growth and in models of $\beta$ cell regeneration. This work adds to mounting evidence that in most physiological and pathological conditions, $\beta$ cell neogenesis may not make large contributions to the postnatal $\beta$ cell pool - at least not in rodents.
\end{abstract}

We have long known that type 1 diabetes results from the autoimmune destruction of $\beta$ cells. More recently, a consensus has developed that the most common form of diabetes, type 2 diabetes, results from the failure of $\beta$ cells to compensate for increased insulin demand, which is associated with the increased calorie intake and decreased exercise that characterizes our modern life. Many factors may contribute to $\beta$ cell failure in type 2 diabetes, but the total number of $\beta$ cells, i.e., $\beta$ cell mass, is clearly one important contributor. At autopsy, $\beta$ cell mass varies substantially among young nondiabetic adults in the population, to a degree that exceeds the variation in height, weight, or BMI (1). Furthermore, $\beta$ cell mass is greater in set-

Conflict of interest: Michael S. German receives research support from Sanofi.

Citation for this article: J Clin Invest. 2013; 123(5):1936-1938. doi:10.1172/JCI69297. tings of increased insulin demand, such as pregnancy $(2,3)$ and obesity (4-8), suggesting some plasticity in the size of our $\beta$ cell pool. Most importantly, all studies to date have demonstrated that patients with type 2 diabetes have reduced $\beta$ cell mass, despite their increased insulin demand (4-9). It seems fairly obvious, therefore, that to understand the pathogenesis of diabetes and develop better therapies, we need to pool of $\beta$ cells, how much capacity we have as adults to generate new $\beta$ cells, and where those $\beta$ cells come from.

\section{Rodent models of $\beta$ cell generation give conflicting answers}

Unfortunately, we cannot measure $\beta$ cell mass in live humans, determine how that mass might change over time, or trace the source of any newly formed $\beta$ cells. Therefore, studies of $\beta$ cell growth and regeneration have turned to animal models, most understand what controls the size of our commonly that of rodents. During fetal development in rodents, $\beta$ cells differentiate from non $-\beta$ cell precursors through a process termed neogenesis (Figure 1). Studies in rodent embryos have worked out the pathways and genes involved in fetal neogenesis of $\beta$ cells (10). A critical step in this process is the decision by pancreatic progenitor cells to adopt an endocrine fate, as opposed to an acinar or duct cell fate. The transcription factor neurogenin 3 (NGN3, also known as Neurog3) controls the endocrine fate decision: its activation in scattered cells within the cords of pancreatic progenitor cells that form the fetal pancreatic ducts is both necessary and sufficient to drive their differentiation into endocrine cells (10). Because NGN3 expression is transient, it also acts as a useful marker of cells in the process of differentiating into endocrine cells, and the abundance of these NGN3-expressing endocrine progenitor cells is often used as a surrogate for the rate of fetal endocrine cell neogenesis.

Fetal neogenesis of $\beta$ cells in rodents stops at birth (11-14), but the newly differentiated $\beta$ cells, which are initially quiescent, start to proliferate rapidly, outstripping the overall growth rate and insulin requirement of the organism (15). This perinatal wave of proliferation also occurs in humans and causes a growth spurt in the $\beta$ cell population that establishes the size of the $\beta$ cell pool prior to the onset of puberty and adulthood (1). Once this wave 